\title{
DISEÑO Y VALIDACIÓN DE UNA BATERÍA DE HABILIDADES MOTRICES BÁSICAS PARA NIÑOS ENTRE 5 Y 11 AÑOS

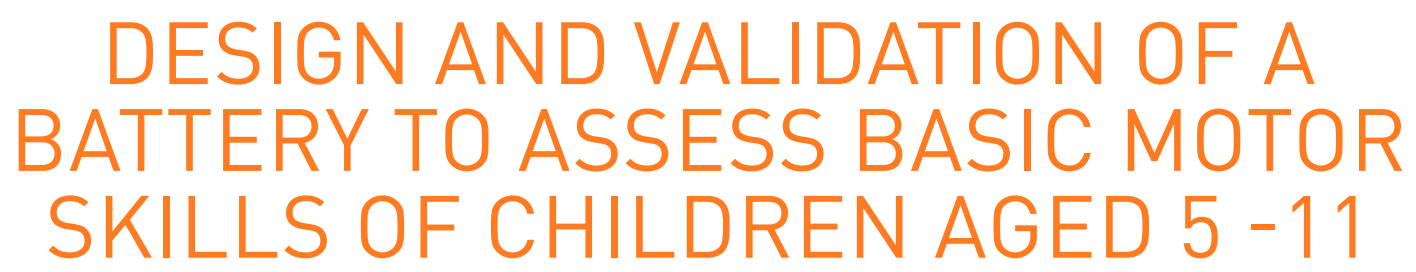

\author{
Enoc Valentín González Palacio \\ Noelva Eliana Montoya Grisales²
}

Yeison Andrés Cardona ${ }^{3}$

Juan Paulo Marín ${ }^{4}$

Bertha Aurora Muñoz ${ }^{5}$

\section{RESUMEN}

La intención de esta investigación fue diseñar y validar una batería sobre habilidades motrices básicas en niños y niñas de 5 a 11 años útil tanto en el contexto escolar como en los procesos de iniciación y formación deportiva. El estudio fue cuantitativo, no experimental descriptivo, el proceso de validación de contenido contó con la participación de 12 expertos en el campo de la educación física escolar, la iniciación y formación

1 Doctor en Educación, docente Universidad de San Buenaventura-Medellín,

https://orcid.org/0000-0001-7708-7886 enoc.gonzalez@usbmed.edu.co. Licenciado en Educación: Educación Física; Especialista en Estadística aplicada; Magister en Motricidad-Desarrollo Humano; Doctor en Educación.

$2 \quad$ Magister en Ciencias de la Educación, docente Universidad de San Buenaventura-Medellín, Noelva Eliana Montoya Grisales: https://orcid.org/0000-0003-3267-6999 noelva.montoya@usbmed.edu.co. Licenciada en Educación Física; Especialista en Educación Física: Actividad Física y Salud; Magister en Ciencias de la Educación

$3 \quad$ Magister en Ciencias de la Educación, docente Universidad de San Buenaventura-Medellín, Yeison Andrés Cardona Molina: https://orcid.org/0000-0002-9185-1665 yeison.cardona@usbmed.edu.co. Licenciada en Educación Física; Magister en Educación

4 Doctor en Ciencias de la Educación, docente Politécnico Jaime Isaza Cadavid, Juan Paulo Marín Castaño: https:// orcid.org/0000-0001-9357-7981

juanmarin@elpoli.edu.co. Licenciado en Educación Física; Magister en Discapacidad; Doctor en Ciencias de la Educación. $5 \quad$ Magister en Educación, docente Politécnico Jaime Isaza Cadavid, Bertha Aurora Muñoz Rodríguez: https://orcid. org/0000-0001-5172-8075

bamunozr@elpoliedu.co. Tecnóloga en Educación Física, Recreación y Deportes; Especialista en Educación Física: Actividad Física y Salud; Magister en Educación- Docencia 
deportiva, el área biomédica y la fisioterapia. La valoración test - retest se hizo con 73 sujetos; en la evaluación interjueces se contó con la presencia de 83 individuos, en ambos casos de género masculino y femenino con edades entre los 5 y 11 años pertenecientes al Valle de Aburrá del departamento de Antioquia - Colombia. La batería se estructuro a partir de 16 indicadores o habilidades motrices, distribuidas en tres componentes: habilidades motrices básicas de locomoción, manipulación y estabilizaciónequilibrio, las cuales presentaron valores muy positivos en la validez de contenido y fiabilidad intra e interjueces. La principal conclusión que se deriva del estudio es ofrecer una batería válida y confiable para la valoración y diagnóstico de las habilidades motrices básicas.

\section{PALABRAS CLAVE}

Evaluación, habilidades motrices básicas, locomoción, manipulación, estabilización, educación física, deporte escolar.

\section{ABSTRACT}

The intention of this research was to design and validate a battery on basic motor skills in boys and girls from 5 to 11 years old, useful both in the school context and in the sports initiation and training processes. The study was quantitative, not descriptive experimental, the content validation process had the participation of 12 experts in the field of school physical education, sports initiation and training, the biomedical area and physiotherapy. The test retest assessment was made with 73 subjects; the inter-judge evaluation included the presence of 83 individuals, both male and female, with ages between 5 and 11 years old, belonging to Valle de Aburrá del departamento de Antioquia - Colombia. The battery was structured from 16 indicators or motor skills, distributed in three components: basic motor skills of locomotion, manipulation and stabilization-balance, which presented very positive values in content validity and intra- and inter-judge reliability. The main conclusion derived from the study is to offer a valid and reliable battery for the assessment and diagnosis of basic motor skills.

\section{KEYWORDS}

Assessment, basic motor skills, locomotion, manipulation, stabilization, physical education, school sports.

\section{INTRODUCCIÓN}

Las habilidades motrices básicas (HMB) se pueden entender como las que son comunes a todas las personas y la base para aprendizajes motores más complejos (Ruiz, 1987); como lo menciona Batalla (2018) son el alfabeto o vocabulario básico de la motricidad, es por ello que se hace importante tener herramientas que permitan evaluar esos elementos cualitativos del movimiento para de esa manera sea posible determinar el estado de desarrollo motriz en el que se encuentra el niño, planificar clases que mejoren las habilidades en aquellos sujetos que muestren retrasos, o potencializar más en aquellos que presentan un adecuado desarrollo.

En el contexto internacional existen diferentes test o baterías que permiten la evaluación de patrones fundamentales de movimiento, en Europa, por ejemplo, existen múltiples publicaciones académicas que evidencian una amplia variedad de estas, entre las más reconocidas están: El Motoriktest für vier- bis sechsjährige Kinder (MOT4-6), es un prueba de origen alemán, tiene como objetivo evaluar el desarrollo motor en niños entre los 4 y 6 años, presenta 17 tareas de prueba (destreza corporal, capacidad de coordinación, habilidades motoras finas y equilibrio) y una actividad de calentamiento, tiene costo para su utilización (Zimmer \& Volkamer, 1987); el inventario de desarrollo de Batelle (Newborg, Stock \& Wnek, 1996) es una batería para evaluar las habilidades fundamentales del desarrollo en niños con 
edades comprendidas entre el nacimiento y los ocho años, validada en España, se aplica de forma individual y está tipificada, está integrado por un total de 341 ítems agrupados en diferentes áreas (Personal/Social, Adaptativa, Motora, Comunicación y Cognitiva), siendo la motora, la encargada de evaluar el control muscular, coordinación corporal, locomoción, motricidad fina y perceptiva y por último, la batería llamada evaluación de las habilidades motrices básicas (Fernández et al., 2007) que permite valorar el grado de adquisición de las habilidades de desplazamiento, giro y manejo de móviles, desarrollada en estudiantes de educación primaria de Madrid.

Para el caso de América entre los más utilizados están: La batería psicomotora propuesta por Da Fonseca (1998), la cual fue diseñada para evaluar niños con dificultades en el aprendizaje a partir de un conjunto de tareas motoras estructuradas en 7 factores psicomotores; el Test of Gross Motor Development versión 2 (TGMD2) identifica déficit en el desarrollo motor grueso evaluando 12 habilidades motrices básicas, fue validado en niños estadounidenses entre los tres y diez años (Ulrich, 2000) y la pauta de observación de patrones motores validado en preescolares Chilenos, la cual posee ocho dimensiones para evaluar marcha, carrera, salto, lanzamiento, golpes y equilibrio (Vargas, 2004).

Para el caso específico de Colombia las herramientas son muy reducidas, la más usual es la escala abreviada de desarrollo para la valoración clínica de desarrollo de los niños menores de siete años versión 3 , la cual tiene como propósito "identificar, de forma temprana, el riesgo de rezagos en el desarrollo de niños y niñas colombianos" (Colombia, Ministerio de Salud y Protección, 2016) y la estructura general contiene los componentes de desarrollo motor grueso, motor fino-adaptativo, lenguaje y personal social. Es importante tener en cuenta que fue diseñada para ser aplicada por personal de la salud y su análisis no resulta ser tan apropiado para el campo de la educación física y su abordaje en la escuela.

Para el caso de la aptitud física (Fuerza, resistencia, velocidad y flexibilidad), ya existe desde 1993 pruebas estandarizadas para escolares colombianos (Jauregui \& Ordoñez, 1993), las cuales no son solo útiles en el campo educativo, sino también en los procesos de iniciación y formación deportiva. En general, existen posibilidades para evaluar habilidades motrices, pero es importante destacar que la mayoría se centran más en el deterioro motriz y déficit motores (Cools et al., 2009) y que para el caso específico de Colombia no se conoce una herramienta que pueda ser implementada en el ámbito escolar y de la iniciación deportiva y que además de cuenta del estado de las habilidades motrices mediante tareas simples y con la suficiente validez estadística.

Por todo lo mencionado anteriormente es que se genera esta batería a partir de criterios de validez y fiabilidad propios del contexto local y como un instrumento de medición de las HMB aplicable a los niños entre 5 y 11 años, posee 16 pruebas distribuidas en tres habilidades: Locomoción con 5 pruebas, manipulación con 8 pruebas y estabilización con 3 pruebas. Los resultados permitirán establecer patrones motores útiles en la valoración y evaluación de niños, convirtiéndose en una herramienta útil para entrenadores, instructores y docentes de la educación física, la recreación, el deporte y la educación infantil.

\section{METODOLOGÍA}

La investigación fue cuantitativa de tipo no experimental descriptiva (Polit y Hungler, 2005), dado que no hubo manipulación de variables independientes, y la intención fue diseñar y validar una batería de pruebas sobre habilidades motrices básicas en niños y niñas en edad 
escolar (5 - 11 años); batería que además es un instrumento que permite caracterizar dicha población en cuanto un componente clave en la valoración y evaluación del desarrollo motor de los infantes en los procesos referidos al deporte escolar, tato dentro y fuera de la escuela.

Para el diseño y construcción de la batería, en términos de validez de contenido se realizó una revisión documental a partir de la propuesta de González Palacio (2019) que permitió conocer a partir de la literatura especializada, cuales son los elementos y posibles dimensiones de la prueba; también se contó con un juicio de expertos para la determinación de la concordancia, coherencia y claridad de los diferentes aspectos de las diferentes pruebas de la batería, por lo cual se tuvieron en cuenta los elementos cuyo CVR (Content Validity Ratio) o Indice de Validez de Contenido sea superior a 0,5823 (reactivos esenciales) de acuerdo a los criterios de TristánLópez (2008) propuesta derivada del modelo de

Para determinar la fiabilidad de la batería y sus componentes se utilizaron los criterios de validez lógica propuesto por Safrit (1981), por cual se realizaron dos procedimientos, una valoración test - retest, por lo que la batería propuesta se aplicó dos veces al mimo grupo de sujetos con una semana de diferencia entre evaluación y otra; el segundo procedimiento consistió en filmar a otro grupo de sujetos y se le pidió a dos expertos en el tema de las habilidades motrices básicas que los evaluara independientemente; por lo tanto la fiabilidad se determinó a partir de un criterio intrajueces y otro interjueces, para determinar la relación entre las variables se usó el coeficiente de correlación intraclase (ICC), un valor de $\mathrm{P}<0,05$ y un intervalo de confianza del $95 \%$.

En este estudio se utilizó un muestreo no probabilístico que implico la escogencia de varias muestras a saber: en el proceso de validación de contenido se escogieron de

manera intencionada 12 jueces expertos que cumplieran con los siguientes requisitos: tener título de maestría o doctorado, con experiencia en la docencia escolar en el área de educación física, el deporte escolar o en procesos de iniciación y formación deportiva; además se incluyeron profesionales en el campo biomédico y la fisioterapia.

En el proceso de valoración intrasujetos se contó con la presencia de 73 niñas y niños con edades entre los 5 y 11 años de los grados de preescolar hasta 6 grado, perteneciente o no a clubes y escuelas deportivas del Valle del Aburra del departamento de Antioquia.

En la evaluación interjueces se tomaron 83 niñas y niños con edades entre los 5 y 11 años de los grados de preescolar hasta 6 grado, perteneciente o no a clubes y escuelas deportivas del Valle del Aburra del departamento de Antioquia.

Los criterios de inclusión tenidos en cuenta fueron: tener entre 5 y 11 años, contar con el consentimiento informado de los padres de familia y el asentimiento por parte de los menores de edad; frente a los criterios de exclusión se precisó: Presentar algún tipo de discapacidad, tener impedimento médico para realizar las pruebas y no completar la totalidad de la batería propuesta.

Para la recolección de la información se aplicó un cuestionario de perfil social y la batería de habilidades motrices básicas; los datos fueron digitados en Excel 2016 y se analizaron en el software SPSS versión 23.

Los instrumentos y procedimientos del estudio fueron sometidos al comité de bioética de la Universidad de San Buenaventura de Medellín, además se tuvieron en cuenta las consideraciones éticas del Informe Helsinki y la Resolución 8430 de 1993 del Ministerio de Salud Nacional de Colombia. 


\section{RESULTADOS}

Para la construcción de la presente batería sobre habilidades motrices básicas inicialmente se realizó una revisión documental sobre la temática, esto con el fin de conocer cuáles debían ser los principales elementos y componentes del instrumento (ítems, indicadores y tipo de habilidades a evaluar), ver tabla 4; los criterios de búsqueda de la revisión fueron los siguientes: habilidades motrices básicas (OR) patrones motores (OR) patrones de movimiento (AND) niños (OR) niñas (NOT) habilidades motrices específicas, en las bases de datos: Google académico, Dialnet, Ebsco y Scielo en el periodo de tiempo comprendido entre $2010 \mathrm{y}$ 2020; en total se encontraron 121 artículos los cuales se sintetizaron en Resúmenes Analíticos de investigación (RAI) (González Palacio, 2019).

De los resultados de la revisión documental se extrajeron 16 habilidades (indicadores) cada una de ellas con una serie de ítems que la determinan (ver tabla 4), éstas habilidades individuales se clasificaron en tres tipos de habilidades motrices básicas: Locomoción, Manipulación y Estabilidad - equilibrio (ver tablas 1 y 4), las cuales se constituyeron en la batería a validar y proponer.
La batería propuesta fue evaluada por 12 expertos en el tema los cuales revisaron los ítems de cada indicador y su pertinencia con la clasificación ofrecida, todos los 16 indicadores o habilidades propuestas para conformar la prueba final fueron aceptados y bien valorados por la mayoría de jueces presentando Razones de Validez de Contenido (CVR) muy por encima del valor de referencia $(0,5823)$ de hecho los valores de CVR oscilaron entre 0,92 y 1,00 (ver tabla 1) que es una valoración muy buena (Tristán-López, 2008).

Los componentes de la batería referidas a habilidades motrices básicas de locomoción, manipulación y estabilidad equilibrio, también obtuvieron calificaciones muy positivas en el Índice de Validez de Contenido (CVI) a saber: 097, 0,99 y 1,00 respectivamente, el CVI de la prueba total fue de 0,98 , que es un resultado que indica un alto grado de consenso entre los jueces que evaluaron la batería propuesta (Tristán-López, 2008) (ver tabla 1).

La batería definitiva se sometió a una prueba piloto con el fin de identificar la comprensión de la prueba por parte de los evaluadores y de las instrucciones por parte de los niños y niñas (15 en total) a los cuales se les aplicó, encontrándose resultados satisfactorios en su realización.

Tabla 1. Resultados de validez de contenido a partir del juicio de expertos.

\begin{tabular}{|c|c|c|c|c|c|}
\hline \multirow{2}{*}{$\begin{array}{c}\text { Clasificación } \\
\text { De } \\
\text { Habilidades }\end{array}$} & \multirow{2}{*}{ Habilidad } & \multicolumn{2}{|c|}{$\begin{array}{c}\text { Evaluación } \\
\text { Expertos }\end{array}$} & \multirow[b]{2}{*}{ CVR } & \multirow[b]{2}{*}{ CVI } \\
\hline & & $A$ & NA & & \\
\hline \multirow[t]{5}{*}{ Locomoción } & Marcha & 11 & 1 & 0,92 & \multirow{5}{*}{0,97} \\
\hline & Correr & 12 & 0 & 1,00 & \\
\hline & $\begin{array}{l}\text { Salto } \\
\text { adelante }\end{array}$ & 11 & 1 & 0,92 & \\
\hline & $\begin{array}{l}\text { Salto en pie } \\
\text { derecho }\end{array}$ & 12 & 0 & 1,00 & \\
\hline & $\begin{array}{l}\text { Salto en pie } \\
\text { izquierdo }\end{array}$ & 12 & 0 & 1,00 & \\
\hline
\end{tabular}




\begin{tabular}{|c|c|c|c|c|c|}
\hline Manipulación & $\begin{array}{l}\text { Lanzamiento } \\
\text { con las } \\
\text { ambas } \\
\text { manos } \\
\text { Lanzamiento } \\
\text { con la mano } \\
\text { derecha } \\
\text { Lanzamiento } \\
\text { con la mano } \\
\text { izquierda } \\
\text { Atrapar } \\
\text { con las dos } \\
\text { manos } \\
\text { Patear con } \\
\text { pie derecho } \\
\text { Patear con } \\
\text { pie izquierdo } \\
\text { Recepción } \\
\text { con el pie } \\
\text { derecho } \\
\text { Recepción } \\
\text { con el pie } \\
\text { izquierdo }\end{array}$ & $\begin{array}{l}12 \\
12 \\
12 \\
12 \\
12 \\
12 \\
12\end{array}$ & $\begin{array}{l}0 \\
0 \\
0 \\
0 \\
0 \\
0\end{array}$ & $\begin{array}{l}1,00 \\
1,00 \\
1,00 \\
1,00 \\
1,00 \\
1,00\end{array}$ & 0,99 \\
\hline $\begin{array}{l}\text { Estabilidad- } \\
\text { equilibrio }\end{array}$ & $\begin{array}{l}\text { Equilibrio } \\
\text { dinámico } \\
\text { Equilibrio } \\
\text { estático en } \\
\text { pie derecho } \\
\text { Equilibrio } \\
\text { estático en } \\
\text { pie izquierdo }\end{array}$ & $\begin{array}{l}12 \\
12 \\
12\end{array}$ & $\begin{array}{l}0 \\
0\end{array}$ & $\begin{array}{l}1,00 \\
1,00 \\
1,00\end{array}$ & 1,00 \\
\hline TOTAL HMB & & & & & 0,98 \\
\hline
\end{tabular}

Para el análisis de fiabilidad se utilizó el procedimiento de validez lógica propuesto por Safrit (1981), lo que implicó dos valoraciones una intra y otra interjueces, para lo cual inicialmente se determinó la normalidad de las variables a relacionar pues el procedimiento estadístico a utilizar, coeficiente de correlación intraclase (ICC) así lo establece; se evaluó la normalidad con la prueba Kolmogorov-Snirnov $(n>50)$ encontrándose normalidad en las variables a estudiar $(P>0,05)$.

Al determinar la relación entre el test y el retest en las Habilidades Motrices Básicas de Locomoción (HMB-L) la concordancia entre las dos mediciones fue muy buena $(I C C=0,972)$ con una significancia estadística muy significativa $\left(\mathrm{P}<0,01 ; \mathrm{IC}_{95}=0,956-0,982\right)$; resultados similares se encontraron en la concordancia entre las 
valoraciones de las Habilidades Motrices Básicas de Manipulación (HMB-M) (ICC=0,955; $\left.\mathrm{P}<0,01 ; \quad \mathrm{IC}_{95}=0,929-0,971\right)$ y las Habilidades Básicas de Estabilidad (HMB-E) (ICC=0,979; $\left.\mathrm{P}<0,01 ; \quad \mathrm{IC}_{95}=0,966-0,987\right)$; frente al resultado

de comparar las dos valoraciones del total de la batería de Habilidades Motrices Básicas (HMB) el resultado en concordancia entre los sujetos fue muy satisfactorio y estadísticamente significativo $\left(I C C=0,966 ; \quad P<0,01 ; \quad I C_{95}=0,947-\right.$ $0,979)$ (ver tabla 2).

Tabla 2.

Valores en el Coeficiente de Correlación Intraclase (ICC) en la valoración test - retest.

\begin{tabular}{|c|c|c|c|c|c|c|c|}
\hline \multicolumn{3}{|c|}{ Estadísticas de elemento } & \multicolumn{5}{|c|}{ Coeficiente de correlación intraclase } \\
\hline \multirow[b]{2}{*}{ Variable } & \multirow[b]{2}{*}{ Media } & \multirow[b]{2}{*}{$\begin{array}{l}\text { Desviación } \\
\text { estándar }\end{array}$} & \multirow[b]{2}{*}{$\begin{array}{l}\text { Correlación } \\
\text { intraclase }\end{array}$} & \multicolumn{2}{|c|}{$\begin{array}{c}\text { Intervalo de } \\
\text { confianza }(95 \%)\end{array}$} & \multicolumn{2}{|c|}{ Prueba F } \\
\hline & & & & $\begin{array}{l}\text { Límite } \\
\text { inferior }\end{array}$ & $\begin{array}{l}\text { Límite } \\
\text { superior }\end{array}$ & Valor & Sig \\
\hline $\mathrm{HMB}$ & & & & & & & \\
\hline locomoción & 22,67 & 1,871 & & & & & \\
\hline $\begin{array}{l}\text { Test } \\
\text { HMB }\end{array}$ & & & 0,972 & 0,956 & 0,982 & 69,684 & 0,000 \\
\hline Locomoción & 22,64 & 1,829 & & & & & \\
\hline Retest & & & & & & & \\
\hline HMB & & & & & & & \\
\hline Manipulación & 30,77 & 3,792 & & & & & \\
\hline $\begin{array}{l}\text { Test } \\
\text { HMB }\end{array}$ & & & 0,955 & 0,929 & 0,971 & 43,469 & 0,000 \\
\hline $\begin{array}{l}\text { Manipulación } \\
\text { Retest }\end{array}$ & 30,95 & 3,775 & & & & & \\
\hline HMB & & & & & & & \\
\hline Estabilidad & 12,07 & 1,619 & & & & & \\
\hline $\begin{array}{l}\text { Test } \\
\text { HMB }\end{array}$ & & & 0,979 & 0,966 & 0,987 & 98,642 & 0,000 \\
\hline $\begin{array}{l}\text { Estabilidad } \\
\text { Retest }\end{array}$ & 12,15 & 1,604 & & & & & \\
\hline Total HMB Test & 65,51 & 5,505 & & & & & \\
\hline $\begin{array}{l}\text { Total HMB } \\
\text { Retest }\end{array}$ & 65,74 & 5,437 & 0,966 & 0,947 & 0,979 & 59,267 & 0,000 \\
\hline
\end{tabular}

$\mathrm{n}=73 ; \mathrm{gl}=72$

En el proceso de evaluación interjueces al relacionar los dos resultados la concordancia entre los dos expertos en el componente de HMB-L el resultado fue muy satisfactorio, pues el ICC tuvo un resultado de 0,956 que además fue estadísticamente significativo $(P<0,01$; $\left.\mathrm{IC}_{95}=0,971-0,974\right)$; para el caso de las HMB-M también fueron muy satisfactorios $($ ICC $=0,959$;
$\left.\mathrm{P}<0,01 ; \quad \mathrm{IC}_{95}=0,938-0,973\right)$ al igual que en las HMB-E (ICC=0,989; $\left.\mathrm{P}<0,01 ; \mathrm{IC}_{95}=0,984-0,993\right)$; en general la concordancia entre los jueces para la batería en general fue muy positiva y estadísticamente importante (ICC $=0,977$; $\left.\mathrm{P}<0,01 ; \mathrm{IC}_{95}=0,965-0,985\right)$. 
Tabla 3.

Valores en el Coeficiente de Correlación Intraclase (ICC) en la valoración interjueces.

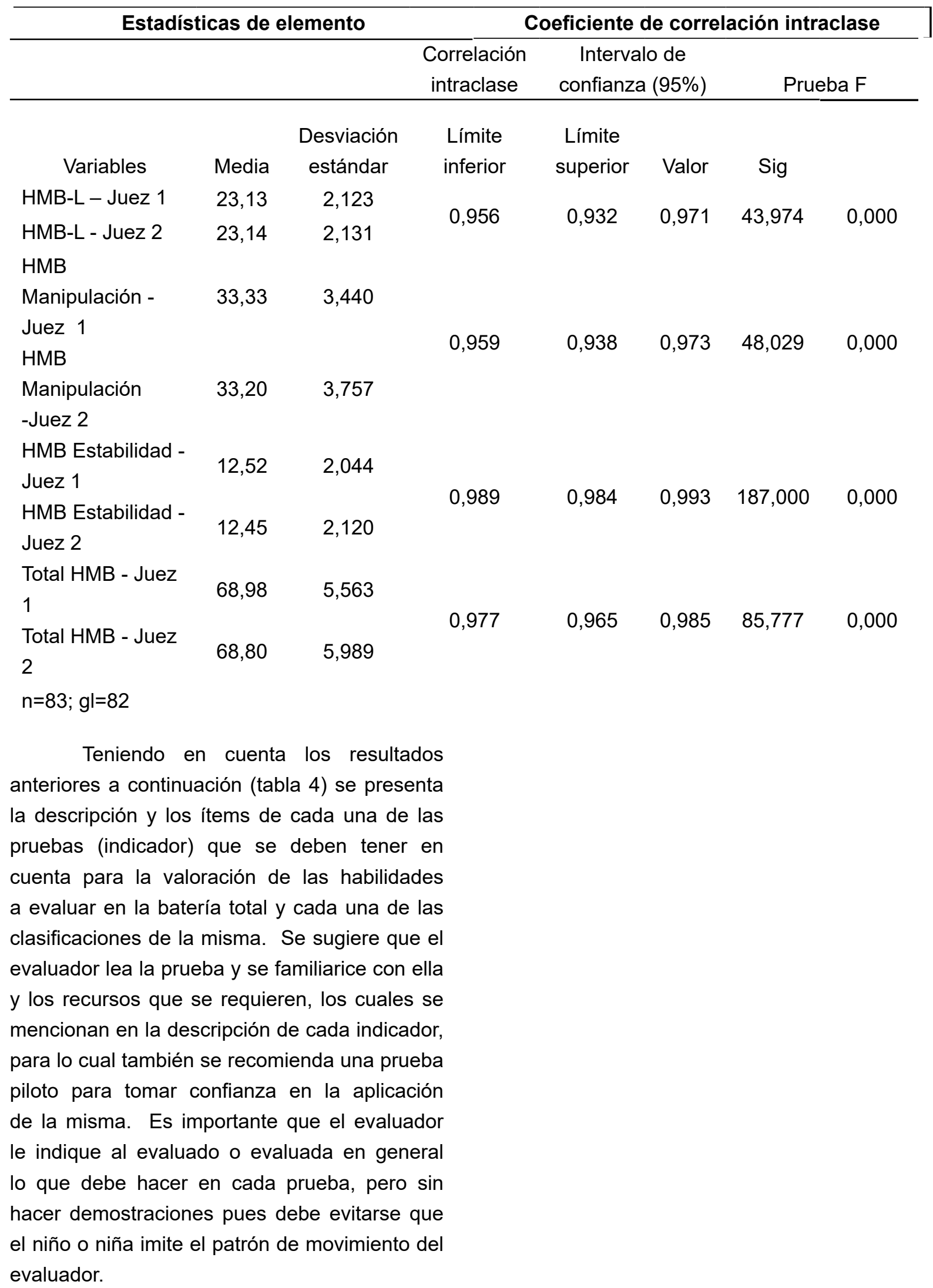


Tabla 4.

Descripción de la batería de habilidades motrices básicas

\begin{tabular}{|c|c|c|c|c|}
\hline $\begin{array}{l}\text { Tipo de } \\
\text { habilidad } \\
\text { motriz }\end{array}$ & $\begin{array}{l}\text { Habilidad- } \\
\text { indicador }\end{array}$ & Descripción & Ítems a tener en cuenta & $\begin{array}{c}\text { Valoración } \\
0 \text { - no lo } \\
\text { hace } \\
1 \text { - lo hace }\end{array}$ \\
\hline \multirow{11}{*}{$Z$} & \multirow{6}{*}{ Marcha } & \multirow{6}{*}{$\begin{array}{l}\text { Cuando el evaluador de } \\
\text { la indicación, el niño } \\
\text { deberá caminar } \\
\text { libremente (desplazarse } \\
\text { hacia adelante } \\
\text { caminando con } \\
\text { calzado), tocar el cono } \\
\text { que se encuentra al } \\
\text { frente y devolverse } \\
\text { hasta el cono de inicio } \\
\text { también caminando } \\
\text { hacia adelante } \\
\text { (distancia entre conos: } \\
9 \text { metros). }\end{array}$} & $\begin{array}{l}\text { *Balanceo libre de los brazos en el plano } \\
\text { sagital y en oposición a las piernas }\end{array}$ & $0-1$ \\
\hline & & & *La posición del tronco es erguida & $0-1$ \\
\hline & & & *Transfiere el peso de talón a punta & $0-1$ \\
\hline & & & $\begin{array}{l}{ }^{*} \text { Existe fase de doble apoyo (en un } \\
\text { momento del desplazamiento ambos } \\
\text { pies tocan el piso) }\end{array}$ & $0-1$ \\
\hline & & & $\begin{array}{l}\text { *Los pies siguen una línea en dirección } \\
\text { al cono }\end{array}$ & $0-1$ \\
\hline & & & TOTAL MARCHA & $0-5$ \\
\hline & \multirow{5}{*}{ Correr } & \multirow{5}{*}{$\begin{array}{l}\text { Cuando el evaluador de } \\
\text { la indicación, el niño } \\
\text { deberá desplazarse } \\
\text { hacia adelante } \\
\text { corriendo (estando } \\
\text { calzado), tocar el cono } \\
\text { que se encuentra al } \\
\text { frente y devolverse } \\
\text { hasta el cono de inicio } \\
\text { (distancia entre conos: } \\
18 \text { metros). }\end{array}$} & $\begin{array}{l}\text { *Los brazos se mueven (adelante - } \\
\text { atrás) describiendo un arco desde los } \\
\text { hombros y están flexionados } \\
\text { aproximadamente } 90^{\circ} \text { y en oposición a } \\
\text { las piernas }\end{array}$ & $0-1$ \\
\hline & & & $\begin{array}{l}{ }^{*} \text { El tronco está levemente inclinado } \\
\text { hacia adelante }\end{array}$ & $0-1$ \\
\hline & & & $\begin{array}{l}\text { *La pierna de apoyo se flexiona en un } \\
\text { movimiento de amortiguación, se acorta } \\
\text { el tiempo de apoyo y se produce una } \\
\text { mayor propulsión }\end{array}$ & $0-1$ \\
\hline & & & $\begin{array}{l}\text { * La pierna que retorna es más } \\
\text { flexionada y el pie se aproxima a los } \\
\text { glúteos }\end{array}$ & $0-1$ \\
\hline & & & $\begin{array}{l}{ }^{*} \text { Breve periodo en el que ambos pies no } \\
\text { tocan el suelo (fase de vuelo) }\end{array}$ & $0-1$ \\
\hline
\end{tabular}




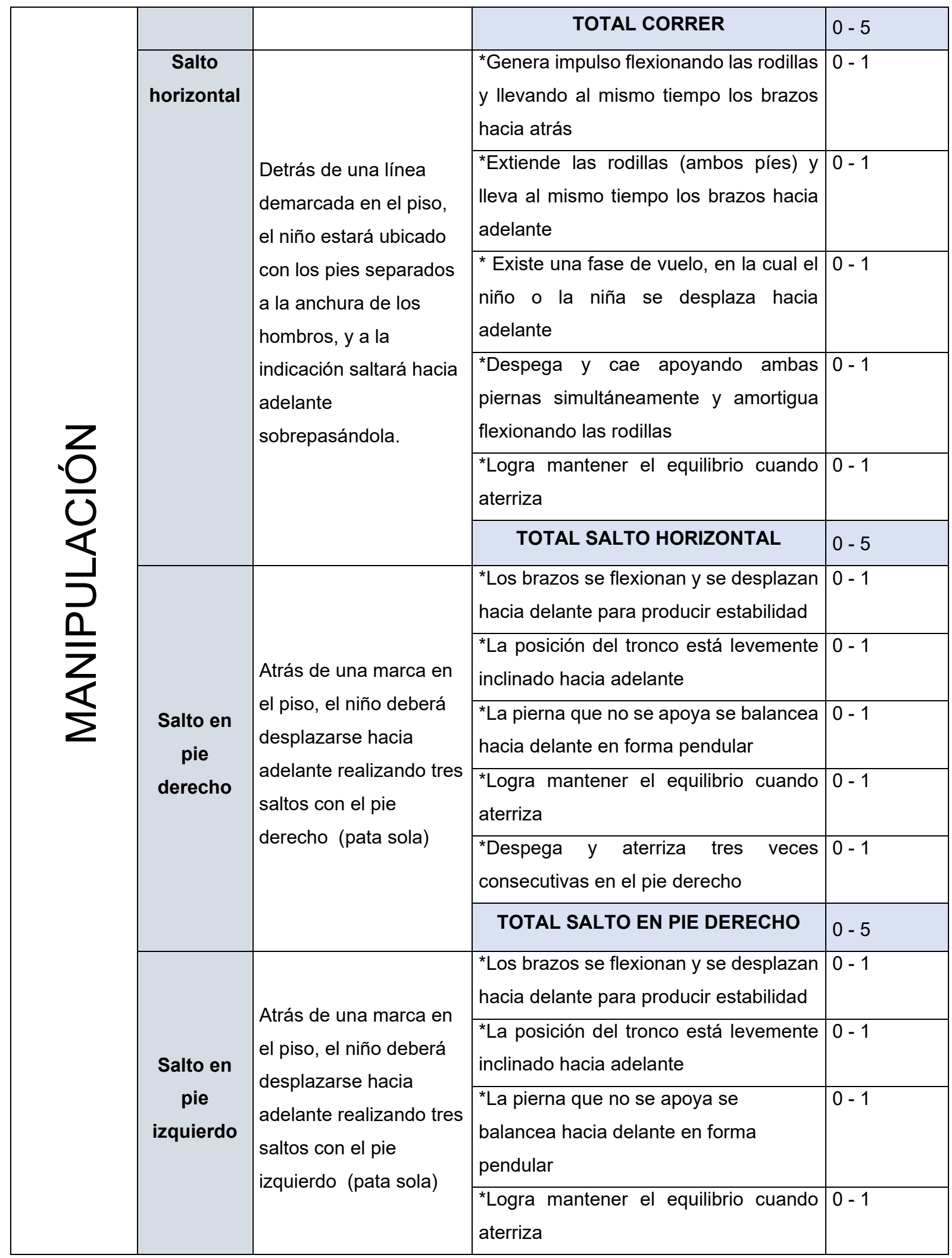




\begin{tabular}{|c|c|c|c|c|}
\hline & & & $\begin{array}{l}{ }^{*} \text { Despega y aterriza tres veces } \\
\text { consecutivas en el pie izquierdo }\end{array}$ & $0-1$ \\
\hline & & & TOTAL SALTO EN PIE IZQUIERDO & $0-5$ \\
\hline \multicolumn{4}{|c|}{ TOTAL LOCOMOCIÓN } & $0-25$ \\
\hline \multirow{12}{*}{$\begin{array}{l}Z \\
\frac{1}{U} \\
\frac{1}{Z} \\
\sum \\
\sum\end{array}$} & \multirow{6}{*}{$\begin{array}{l}\text { Lanzamien } \\
\text { to con las } \\
\text { dos manos }\end{array}$} & \multirow{6}{*}{$\begin{array}{l}\text { El niño con los pies a la } \\
\text { anchura de los hombros } \\
\text { debe situarse a cinco } \\
\text { metros de un aro (o } \\
\text { punto de referencia), } \\
\text { que deberá estar } \\
\text { ubicado a una altura de } \\
\text { un metro y medio en } \\
\text { relación con el piso. } \\
\text { Este deberá lanzar con } \\
\text { ambas manos sobre el } \\
\text { nivel de sus hombros y } \\
\text { por encima de su } \\
\text { cabeza, una pelota de } \\
\text { plástico e intentar } \\
\text { golpear o darle al } \\
\text { centro del aro. }\end{array}$} & $\begin{array}{l}\text { *El lanzamiento se realiza por encima de } \\
\text { la cabeza }\end{array}$ & $0-1$ \\
\hline & & & $\begin{array}{l}\text { *Hay transferencia de movimiento desde } \\
\text { los pies hacia el tronco }\end{array}$ & $0-1$ \\
\hline & & & $\begin{array}{l}\text { *Adelanta una pierna en relación a la } \\
\text { otra cuando inicia el gesto }\end{array}$ & $0-1$ \\
\hline & & & ${ }^{*}$ Existe control de la pelota & $0-1$ \\
\hline & & & $\begin{array}{l}\text { *La pelota avanza hacia el frente (aro o } \\
\text { punto de referencia) }\end{array}$ & $0-1$ \\
\hline & & & $\begin{array}{c}\text { TOTAL LANZAMIENTO CON LAS } \\
\text { DOS MANOS }\end{array}$ & $0-5$ \\
\hline & \multirow{6}{*}{$\begin{array}{c}\text { Lanzamien } \\
\text { to con la } \\
\text { mano } \\
\text { derecha }\end{array}$} & \multirow{6}{*}{$\begin{array}{l}\text { El niño debe situarse } \\
\text { con ambos pies a la } \\
\text { anchura de los hombros } \\
\text { a cinco metros de un } \\
\text { aro (o punto de } \\
\text { referencia), que deberá } \\
\text { estar ubicado a una } \\
\text { altura de un metro y } \\
\text { medio en relación con } \\
\text { el piso. El niño deberá } \\
\text { lanzar con la mano } \\
\text { derecha una pelota de } \\
\text { tenis hacia adelante, a } \\
\text { nivel de sus hombros y }\end{array}$} & $\begin{array}{l}\text { *Extensión total del brazo en el } \\
\text { momento de soltar la pelota. }\end{array}$ & $0-1$ \\
\hline & & & ${ }^{*}$ Rotación del tronco hacia la derecha. & $0-1$ \\
\hline & & & *Tiene la pierna izquierda adelantada & $0-1$ \\
\hline & & & ${ }^{*}$ Existe control de la pelota & $0-1$ \\
\hline & & & $\begin{array}{l}\text { *La pelota avanza hacia el frente (aro o } \\
\text { punto de referencia) }\end{array}$ & $0-1$ \\
\hline & & & $\begin{array}{c}\text { TOTAL LANZAMIENTO CON LA } \\
\text { MANO DERECHA }\end{array}$ & $0-5$ \\
\hline
\end{tabular}




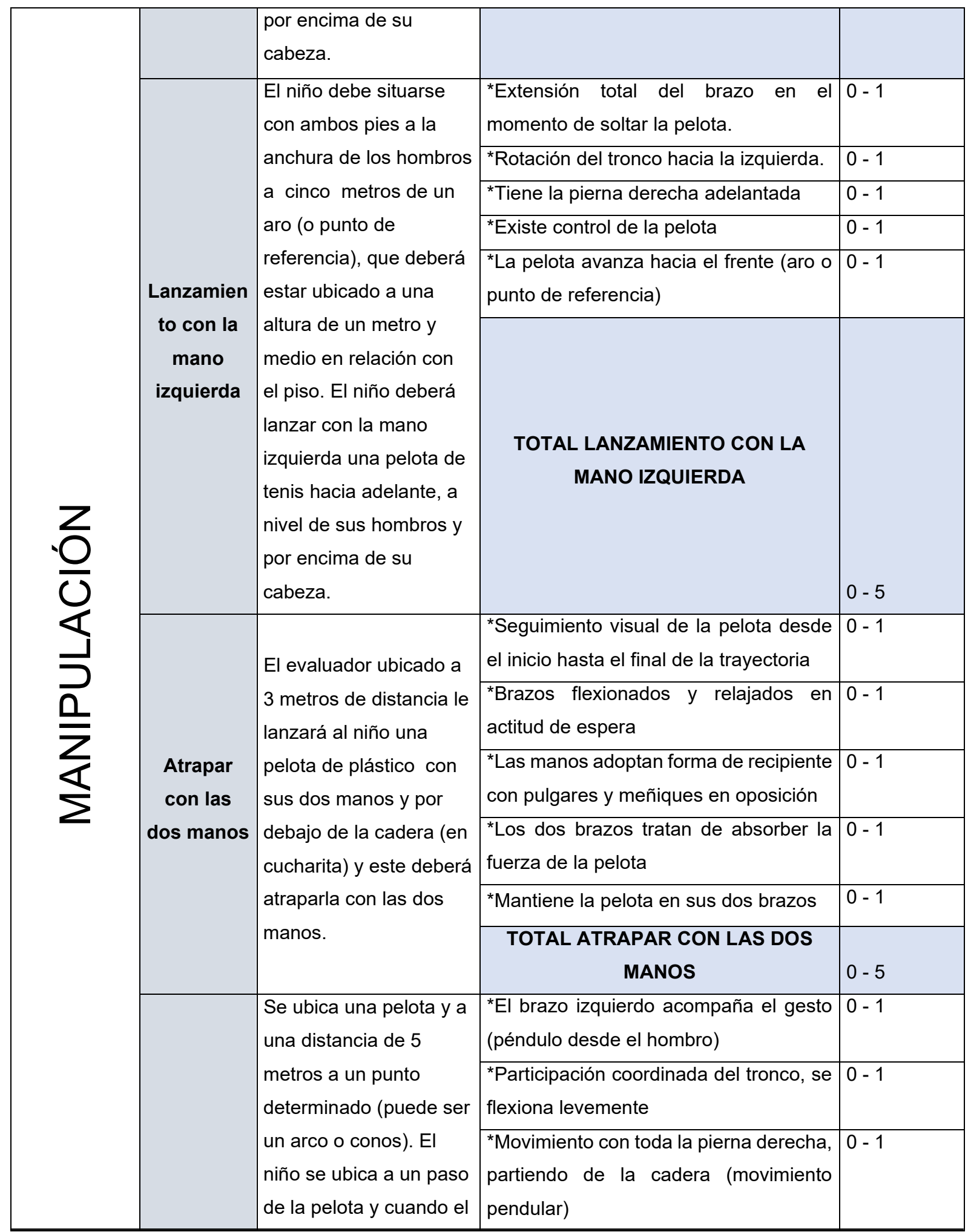




\begin{tabular}{|c|c|c|c|}
\hline \multirow{3}{*}{$\begin{array}{l}\text { Patear con } \\
\text { pie } \\
\text { derecho }\end{array}$} & \multirow{3}{*}{$\begin{array}{l}\text { evaluador de la orden el } \\
\text { niño debe patear la } \\
\text { pelota con el pie } \\
\text { derecho hacia el punto } \\
\text { determinado (puede } \\
\text { usar cualquier } \\
\text { superficie de contacto } \\
\text { del pie: borde interno, } \\
\text { borde externo, punta o } \\
\text { empeine). }\end{array}$} & $\begin{array}{l}\text { *La pierna que ejecuta la acción } \\
\text { (derecha) retorna al punto de partida }\end{array}$ & $0-1$ \\
\hline & & $\begin{array}{l}\text { *Golpea la pelota y esta avanza hacia el } \\
\text { frente (arco o punto de referencia) }\end{array}$ & $0-1$ \\
\hline & & TOTAL PATEAR CON PIE DERECHO & $0-5$ \\
\hline \multirow{6}{*}{$\begin{array}{l}\text { Patear con } \\
\text { pie } \\
\text { izquierdo }\end{array}$} & \multirow{6}{*}{$\begin{array}{l}\text { Se ubica una pelota y a } \\
\text { una distancia de } 5 \\
\text { metros a un punto } \\
\text { determinado (puede ser } \\
\text { un arco o conos). El } \\
\text { niño se ubica a un paso } \\
\text { de la pelota y cuando el } \\
\text { evaluador de la orden, } \\
\text { el niño debe patear la } \\
\text { pelota con el pie } \\
\text { izquierdo hacia el punto } \\
\text { determinado (puede } \\
\text { usar cualquier } \\
\text { superficie de contacto } \\
\text { del pie: borde interno, } \\
\text { borde externo, punta o } \\
\text { empeine). }\end{array}$} & $\begin{array}{l}{ }^{*} \text { El brazo derecho acompaña el gesto } \\
\text { (péndulo desde el hombro) }\end{array}$ & $0-1$ \\
\hline & & $\begin{array}{l}\text { *Participación coordinada del tronco, se } \\
\text { flexiona levemente }\end{array}$ & $0-1$ \\
\hline & & 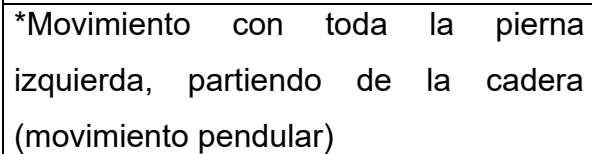 & $0-1$ \\
\hline & & $\begin{array}{l}\text { *La pierna que ejecuta la acción } \\
\text { (izquierda) retorna al punto de partida }\end{array}$ & $0-1$ \\
\hline & & $\begin{array}{l}\text { *Golpea la pelota y esta avanza hacia el } \\
\text { frente (arco o punto de referencia) }\end{array}$ & $0-1$ \\
\hline & & $\begin{array}{l}\text { TOTAL PATEAR CON PIE } \\
\text { IZQUIERDO }\end{array}$ & $0-5$ \\
\hline \multirow{5}{*}{$\begin{array}{l}\text { Recepción } \\
\text { con el pie } \\
\text { derecho }\end{array}$} & \multirow{5}{*}{$\begin{array}{l}\text { El evaluador ubicado a } \\
3 \text { metros de distancia le } \\
\text { lanzará al niño una } \\
\text { pelota de plástico con } \\
\text { sus dos manos por el } \\
\text { piso (de forma rastrera) } \\
\text { y este deberá } \\
\text { recepcionarla con su } \\
\text { pie derecho (puede } \\
\text { usar cualquier }\end{array}$} & $\begin{array}{l}\text { *Seguimiento visual de la pelota desde } \\
\text { el inicio hasta el final de la trayectoria }\end{array}$ & $0-1$ \\
\hline & & ${ }^{*}$ Controla la pelota con su pie derecho & $0-1$ \\
\hline & & $\begin{array}{l}{ }^{*} \text { Controla y mantiene el equilibrio } \\
\text { mientras recibe }\end{array}$ & $0-1$ \\
\hline & & *La pelota no rebota mas de $50 \mathrm{~cm}$ & $0-1$ \\
\hline & & $\begin{array}{c}\text { TOTAL RECEPCIÓN CON EL PIE } \\
\text { DERECHO }\end{array}$ & $0-4$ \\
\hline
\end{tabular}




\begin{tabular}{|c|c|c|c|c|}
\hline & & $\begin{array}{l}\text { superficie de contacto } \\
\text { del pie: borde interno, } \\
\text { borde externo, punta o } \\
\text { empeine). }\end{array}$ & & \\
\hline & \multirow{5}{*}{$\begin{array}{l}\text { Recepción } \\
\text { con el pie } \\
\text { izquierdo }\end{array}$} & \multirow{5}{*}{$\begin{array}{l}\text { El evaluador ubicado a } \\
3 \text { metros de distancia le } \\
\text { lanzará al niño una } \\
\text { pelota de plástico con } \\
\text { sus dos manos por el } \\
\text { piso (de forma rastrera) } \\
\text { y este deberá } \\
\text { recepcionarla con su } \\
\text { pie izquierdo (puede } \\
\text { usar cualquier } \\
\text { superficie de contacto } \\
\text { del pie: borde interno, } \\
\text { borde externo, punta o } \\
\text { empeine). }\end{array}$} & $\begin{array}{l}\text { *Seguimiento visual de la pelota desde } \\
\text { el inicio hasta el final de la trayectoria }\end{array}$ & $0-1$ \\
\hline & & & ${ }^{*}$ Controla la pelota con su pie izquierdo & $0-1$ \\
\hline & & & $\begin{array}{l}{ }^{*} \text { Controla y mantiene el equilibrio } \\
\text { mientras recibe }\end{array}$ & $0-1$ \\
\hline & & & *La pelota no rebota mas de $50 \mathrm{~cm}$ & $0-1$ \\
\hline & & & $\begin{array}{l}\text { TOTAL RECEPCIÓN CON EL PIE } \\
\text { IZQUIERDO }\end{array}$ & $0-4$ \\
\hline \multicolumn{4}{|c|}{ TOTAL MANIPULACIÓN } & $0-38$ \\
\hline & \multirow{6}{*}{$\begin{array}{l}\text { Equilibrio } \\
\text { dinámico }\end{array}$} & \multirow{6}{*}{$\begin{array}{l}\text { El niño se ubican sobre } \\
\text { una línea de } 5 \mathrm{~cm} \text { de } \\
\text { ancho por } 9 \mathrm{~m} \text { de largo } \\
\text { y se le pide que camine } \\
\text { sobre ésta línea hasta } \\
\text { el final del recorrido. }\end{array}$} & *La mirada al frente & $0-1$ \\
\hline & & & ${ }^{*}$ Mantiene una postura erguida & $0-1$ \\
\hline & & & $\begin{array}{l}\text { *Los brazos se coordinan con los pies } \\
\text { contrarios, se mueven de adelante hacia } \\
\text { atrás sin elevarlos lateralmente. }\end{array}$ & $0-1$ \\
\hline & & & $\begin{array}{l}{ }^{*} \text { En el desplazamiento no se inclina a los } \\
\text { lados }\end{array}$ & $0-1$ \\
\hline & & & $\begin{array}{l}\text { *Los pies se mantienen todo el tiempo } \\
\text { en la línea de la trayectoria }\end{array}$ & $0-1$ \\
\hline & & & TOTAL EQUILIBRIO DINÁMICO & $0-5$ \\
\hline & \multirow[b]{3}{*}{$\begin{array}{l}\text { Equilibrio } \\
\text { estático en }\end{array}$} & \multirow{3}{*}{$\begin{array}{l}\text { Con la vista al frente, el } \\
\text { niño deberá pararse } \\
\text { descalzo sobre una } \\
\text { colchoneta en el pie } \\
\text { derecho, y con la otra } \\
\text { pierna mantendrá la } \\
\text { rodilla hacia el frente y }\end{array}$} & $\begin{array}{l}\text { *Los brazos se encuentran relajados a } \\
\text { los lados del cuerpo }\end{array}$ & $0-1$ \\
\hline & & & $\begin{array}{l}\text { *Mantiene posición erguida evitando } \\
\text { inclinar el cuerpo adelante - atrás. }\end{array}$ & $0-1$ \\
\hline & & & $\begin{array}{l}\text { *Mantiene posición erguida evitando } \\
\text { inclinar el cuerpo de un lado hacia el otro } \\
\text { (derecha - izquierda o viceversa). }\end{array}$ & $0-1$ \\
\hline
\end{tabular}




\begin{tabular}{|c|c|c|c|}
\hline \multirow[t]{3}{*}{$\begin{array}{c}\text { pie } \\
\text { derecho }\end{array}$} & \multirow{3}{*}{$\begin{array}{l}\text { el talón orientado hacia } \\
\text { atrás (duración } 5 \\
\text { segundos). }\end{array}$} & $\begin{array}{l}\text { *La pierna derecha se mantiene } \\
\text { extendida }\end{array}$ & $0-1$ \\
\hline & & $\begin{array}{l}\text { *La pierna izquierda mantienen la rodilla } \\
\text { delante del cuerpo (posición anterior) y } \\
\text { el talón detrás del cuerpo (posición } \\
\text { posterior) (Plano frontal) }\end{array}$ & $0-1$ \\
\hline & & $\begin{array}{c}\text { TOTAL EQUILIBRIO ESTÁTICO EN } \\
\text { PIE DERECHO }\end{array}$ & $0-5$ \\
\hline \multirow{6}{*}{$\begin{array}{l}\text { Equilibrio } \\
\text { estático en } \\
\text { pie } \\
\text { izquierdo }\end{array}$} & \multirow{6}{*}{$\begin{array}{l}\text { Con la vista al frente, el } \\
\text { niño deberá pararse } \\
\text { descalzo sobre una } \\
\text { colchoneta en el pie } \\
\text { izquierdo, y con la otra } \\
\text { pierna mantendrá la } \\
\text { rodilla hacia el frente y } \\
\text { el talón orientado hacia } \\
\text { atrás (duración } 5 \\
\text { segundos). }\end{array}$} & $\begin{array}{l}\text { *Los brazos se encuentran relajados a } \\
\text { los lados del cuerpo }\end{array}$ & $0-1$ \\
\hline & & $\begin{array}{l}\text { *Mantiene posición erguida evitando } \\
\text { inclinar el cuerpo adelante - atrás. }\end{array}$ & $0-1$ \\
\hline & & $\begin{array}{l}\text { *Mantiene posición erguida evitando } \\
\text { inclinar el cuerpo de un lado hacia el otro } \\
\text { (derecha - izquierda o viceversa). }\end{array}$ & $0-1$ \\
\hline & & $\begin{array}{l}\text { *a pierna izquierda se mantiene } \\
\text { extendida }\end{array}$ & $0-1$ \\
\hline & & $\begin{array}{l}\text { *La pierna derecha mantienen la rodilla } \\
\text { delante del cuerpo (posición anterior) y } \\
\text { el talón detrás del cuerpo (posición } \\
\text { posterior) (Plano frontal) }\end{array}$ & $0-1$ \\
\hline & & $\begin{array}{c}\text { TOTAL EQUILIBRIO ESTÁTICO EN } \\
\text { PIE IZQUIERDO }\end{array}$ & $0-5$ \\
\hline & \multicolumn{2}{|c|}{ TOTAL ESTABILIDAD-EQUILIBRIO } & $0-15$ \\
\hline & \multicolumn{2}{|c|}{ SUMATORIA TOTAL DE LAS HMB } & 78 \\
\hline
\end{tabular}

\section{CONCLUSIONES}

En términos generales la más importante conclusión que se deriva del estudio es que se presenta una batería que evalúa el perfil de habilidades motrices básicas que es válida y fiable para un contexto colombiano, los valores encontrados en términos de validez de contenido $y$ de fiabilidad intra e interjueces fueron muy positivos y estadísticamente significativos lo que hace que el instrumento propuesto sea una herramienta útil en el contexto de la educación física escolar y los procesos de iniciación y formación deportiva.

La batería propuesta permite evaluar el perfil total de habilidades motrices básicas, pero también es susceptible de usarse de manera específica, es decir, es posible utilizarse para evaluar únicamente el perfil de habilidades motrices básicas de locomoción, manipulación o estabilización dependiendo de las necesidades o interés del profesor de educación física, entrenador o investigador.

La valoración de las habilidades motrices básicas no solo radica en su diagnóstico y descripción, pues además su adecuado desarrollo implica tener una base para el desarrollo de habilidades más complejas (Ruiz, 1987); de hecho, como establece Batalla (2018) las habilidades motrices básicas son el fundamento básico de la motricidad, y sus implicaciones están relacionados en el campo del deporte, las actividades artísticas, la salud y la vida cotidiana. 
El conjunto de pruebas validadas en este texto se convierte en un instrumento útil para el diagnóstico de patrones básicos de movimiento en niños y niñas entre los 5 y 11 años, lo cual es relevante en términos del desarrollo motor y perfil de salud de los infantes, además es una herramienta útil para el desarrollo de clase en el área de educación física, recreación y deporte escolar. Por otro lado, es un insumo para investigadores en el campo del desarrollo motor en niños y niñas especialmente en lo concerniente a habilidades motrices básicas.

La evaluación en el campo de la educación física ha sido un elemento controversial y multidimensional (González Palacio et al, 2020), que ha implicado elementos desde lo conceptual, procedimental y actitudinal, pero sobre todo la valoración de los estudiantes, esto hace que esta batería sea una de las tantas formas de aportar a este proceso al interior de la escuela y fuera de ella.

\section{REFERENCIAS BIBLIOGRÁFICAS}

Batalla Flores, A. (2018). Habilidades motrices (2 ed.). Barcelona: INDE

Colombia, Ministerio de Salud y Protección. (2016). Escala abreviada de desarrollo-3. Ministerio de Salud y Protección. https:// cutt.ly/xh0lzM8

Colombia. Ministerio de Salud Nacional. (1993). Resolución número 8430 de 1993. Por la cual se establecen las normas científicas, técnicas y administrativas para la investigación en salud. Diario Oficial. https://cutt.ly/yfYs1G6.

Cools, W., De Martelaer, K., Samaey, C. \&Andries, C. (2009). Movement skill assessment of typically developing preschool children: a review of seven movement skill assessment tools. J Sports Sci Med, 8(2),154-68
Da Fonseca, V. (1998). Manual de observación psicomotriz. Factores psicomotores y su relación con las tres unidades funcionales: Fundamentos psiconeurológicos de la batería psicomotora (BPM). INDE.

Fernández, E., Gardoqui, M.L. \& Sánchez, F. (2007). Evaluación de las habilidades motrices básicas. INDE

GonzálezPalacio, E. V.(2019). Representaciones sociales sobre la formación inicial y ser maestro en estudiantes de educación física del departamento de Antioquia. [Tesis de doctorado, Universidad de Antioquia]. Biblioteca digital UdeA. http://hdl.handle.net/10495/13905

González Palacio, E., Chaverra Fernández, B., Bustamante Castaño, S., \& Toro Suaza, C. (2020). Diseño y validación de un cuestionario sobre las concepciones y percepción de los estudiantes sobre la evaluación en Educación Física. Retos, (40), 317-325. https://doi.org/10.47197/ retos.v1i40.80914

Jauregui, G., \& Ordoñez, O. (1993). Aptitud física: pruebas estandarizadas en Colombia. Coldeportes Nacional.

Newborg, J., Stock, J. R. \& Wnek, L. (1996). Inventario de Desarrollo Batelle. TEA.

Polit, H. \& Hungler, B. (2005). Investigación científica en ciencias de la salud (6. ed.). McGraw-Hill.

Ruiz, L. M. (1987). Desarrollo Motor y Actividad Física. Gymnos.

Safrit, M. (1981). Evaluation in Physical Education. Prentice-Hall.

Tristán-López, A. (2008). Modificación al modelo de Lawshe para el dictamen cuantitativo de la validez de contenido 
de un instrumento objetivo. Avances en Medición, 6(1), 37-48. https://cutt.ly/ thOIQgv

Ulrich, D.A. (2000). TGMD-2: Test of Gross Motor Development (2.ed.). Pro-ed.

Vargas, C. R. (2004). Pauta de Observación de los patrones motores en niños de 4 a 6 años: diseño, validación, observación del desarrollo motor $y$ propuesta de estimulación. [Tesis de doctorado]. Universidad de Valladolid, Facultad de Educación y Trabajo Social, Departamento de Pedagogía, Valladolid.

Zimmer, R. \& Volkamer, M. (1987). Motoriktest für vier- bis sechsjärige Kinder (manual). Weinheim. 